BMJ Open

Diabetes

Research

$\&$ Care

\title{
Lipid accumulation product (LAP) as a potential index to predict risk of insulin resistance in young, non-obese Asian Indian males from Southern India: observations from hyperinsulinemic- euglycemic clamp studies
}

\author{
Shajith Anoop S, ${ }^{1}$ Riddhi Dasgupta (D) , ${ }^{1}$ Grace Rebekah, ${ }^{2}$ Arun Jose, ${ }^{3}$ \\ Mercy Prem Inbakumari, ${ }^{1}$ Geethanjali Finney, ${ }^{1}$ Nihal Thomas (D) ${ }^{3}$
}

To cite: Anoop S S, Dasgupta R, Rebekah G, et al. Lipid accumulation product (LAP) as a potential index to predict risk of insulin resistance in young, nonobese Asian Indian males from Southern India: observations from hyperinsulinemiceuglycemic clamp studies. BMJ Open Diab Res Care 2021;9:e002414. doi:10.1136/ bmjdrc-2021-002414

Received 4 June 2021 Accepted 15 August 2021

Check for updates

(C) Author(s) (or their employer(s)) 2021. Re-use permitted under CC BY-NC. No commercial re-use. See rights and permissions. Published by BMJ.

${ }^{1}$ Department of Endocrinology, Diabetes and Metabolism, Christian Medical College and Hospital Vellore, Vellore, Tamil Nadu, India

${ }^{2}$ Biostatistics, Christian Medical College and Hospital Vellore, Vellore, Tamil Nadu, India ${ }^{3}$ Department of Clinical Biochemistry, Christian Medical College and Hospital Vellore, Vellore, Tamil Nadu, India

Correspondence to Dr Riddhi Dasgupta; riddhi_dg@rediffmail.com

\section{ABSTRACT}

Introduction We aimed to compare the predictive accuracy of surrogate indices namely the lipid accumulation product (LAP) index, homeostatic model of assessment of insulin resistance (HOMA-IR), fasting glucose-insulin ratio (FG-IR) and the quantitative-insulin sensitivity check index (QUICKI), against the $M$ value of hyperinsulinemic-euglycemic clamp (HEC), and to determine a cut-off value for the LAP index to predict risk of insulin resistance in non-obese (body mass index $<21 \mathrm{~kg} / \mathrm{m}^{2}$ ), normoglycemic, Asian Indian males from Southern India.

Research design and methods Data of HEC studies performed in 108 non-obese, normoglycemic, Asian Indian males was obtained retrospectively and the $M$ value (a measure of whole-body insulin sensitivity) was calculated. The $M$ value is the rate of whole-body glucose metabolism at the hyperinsulinemic plateau (a measure of insulin sensitivity) and is calculated between 60 and $120 \mathrm{~min}$ after the start of the insulin infusion in the HEC procedure. The LAP index, the HOMA-IR, FG-IR and QUICKI were calculated. Spearman's correlation and logistic regression analysis were performed. Cut-off value for the LAP index was obtained using receiver operating characteristics with area under curve (AUC) analysis at $95 \% \mathrm{Cl}$. P value $<0.05$ was considered to be statistically significant.

Results Significant negative correlation was observed for the $M$ value with LAP index $(r=-0.39, p<0.001)$ while significant positive correlation was noted with FG-IR $(r=0.25 ; p<0.01)$ and QUICKI $(r=0.22 ; p<0.01)$. The LAP index cut-off value $\geq 33.4$ showed $75 \%$ sensitivity and $75 \%$ specificity with AUC (0.72) to predict risk of insulin resistance in this cohort.

Conclusion The LAP index showed higher predictive accuracy for the risk of insulin resistance as compared with HOMA-IR, QUICKI and FG-IR in non-obese, normoglycemic Asian Indian males from Southern India.

\section{INTRODUCTION}

The global burden of non-communicable diseases is driven majorly by type 2 diabetes

\section{Significance of this study}

What is already known about this subject?

- Surrogate indices of fasting insulin resistance such as homeostatic model of assessment of insulin resistance (HOMA-IR), fasting glucose-insulin ratio (FG-IR) and the quantitative-insulin sensitivity check index (QUICKI) have been validated against the $M$ value of hyperinsulinemic-euglycemic clamp (HEC) studies in various ethnic groups; however, triglyceride-based surrogate indices have been scarely validated against the HEC studies, especially in Asian Indians.

What are the new findings?

- In this study, we have demonstrated the lipid accumulation product (LAP) index to have superior value to predict the future risk of insulin resistance in nonobese, normoglycemic (BMl $\left.<21 \mathrm{~kg} / \mathrm{m}^{2}\right)$ Asian Indian men.

- The cost of screening the LAP index is less when compared with HOMA-IR, QUICKI and FG-IR, as the LAP index relies on waist circumference and serum triglyceride values.

How might these results change the focus of research or clinical practice?

- The LAP index can be used as a cost-effective surrogate index to screen for insulin resistance in clinical settings and epidemiological settings.

mellitus (T2DM), cardiovascular diseases, cancers, stroke, chronic respiratory diseases. ${ }^{1}$ South Asians, especially Asian Indians feature a unique phenotype characterized by increased body fat, less muscle mass and increased abdominal fat, ${ }^{2}$ even at low body mass index (BMI). ${ }^{3}$ This phenotype predisposes to insulin resistance, T2DM and cardiovascular diseases. 
The hyperinsulinemic-euglycemic glucose clamp (HEC) procedure is universally accepted as the 'gold standard' reference method to measure insulin sensitivity as it measures whole body glucose disposal at a given level of insulinemia under steady-state conditions. ${ }^{4}$ However, the HEC procedure is expensive, requires trained scientific manpower and vigilant medical supervision, thus making it impractical for use in large epidemiological and clinical studies. Surrogate indices of fasting insulin resistance such as the quantitative-insulin sensitivity check index (QUICKI), homeostasis model assessment of insulin resistance (HOMA-IR), fasting glucose-insulin ratio (FGIR) have been applied in population-based studies across different ethnic groups. ${ }^{5}$ We have previously shown HOMA-IR is less reliable when validated against $M$ value of HEC procedure in non-obese (BMI $<23 \mathrm{~kg} / \mathrm{m}^{2}$ ) Asian Indian males, thereby emphasizing the need for a better surrogate index of insulin resistance in non-obese Asian Indians. ${ }^{6}$ Surrogate indices such as HOMA-IR, QUICKI and FG-IR are based on fasting insulin levels. The cost for an insulin assay is expensive, thereby making insulinbased surrogate indices less feasible in resource-limited clinical settings and epidemiological studies with large sample sizes. Alternatively, lipid-based surrogate indices of fasting insulin resistance have garnered much interest. One such index is the lipid accumulation product (LAP), which is an ordinal index of insulin resistance derived from one anthropometric variable, that is, waist circumference and one biochemical variable namely serum triglycerides. It was first applied in the National Health and Nutrition Examination Survey sample database as a superior measure of cardiovascular risk in comparison to BMI, in a cohort of non-Hispanic blacks and Mexican Americans. ${ }^{7}$

A recent HEC-based study on Italian subjects with varying degrees of insulin resistance had demonstrated that the LAP index had higher predictive accuracy for individuals with increased vascular stiffness, when compared with triglyceride (TG)/high-density lipoprotein ratio, TG-glucose index and visceral adiposity index (VAI). ${ }^{8}$ Another HEC-based study in Chinese women compared the LAP index, VAI, waist circumference, BMI, HOMA-IR and the Chinese VAI. The study reported that in Chinese women, the Chinese VAI was strongly associated with the $M$ value of HEC procedure and also outperformed the VAI, HOMA-IR, waist circumference and BMI. ${ }^{9}$

A few studies on the LAP index in Asian Indians have shown higher predictive accuracy of the LAP index for insulin resistance. ${ }^{10-12}$ However, such observations have not been correlated with the HEC procedure, which is the gold standard method to determine hepatic and peripheral insulin resistance in Asian Indians. While glucose-based and insulin-based surrogate indices have been validated against the HEC procedure, such an attempt has not been made for the LAP index in any study from India. We hypothesized that the LAP index may be useful to predict future risk of insulin resistance in normoglycemic Asian Indians. Therefore, the primary objective of this study was to correlate the LAP index and other surrogate indices namely HOMA-IR, QUICKI and FG-IR with the $M$ value derived from HEC clamp studies. The secondary objective was to derive significant determinants of the LAP index and to determine a cut-off value for the LAP index to predict insulin resistance in a cohort of non-obese, normoglycemic Asian Indian males from Southern India.

\section{METHODOLOGY}

The study was approved after review by the institutional research Board and human ethics committee of Christian Medical College, Vellore, India (Research Committee Minute Number: 13348/RETRO/28/08/2020 of Christian Medical College, Vellore, India). Data for this study were obtained retrospectively from the primary study based on HEC procedures in normoglycemic, Asian Indian males from Southern India. ${ }^{13}$ The sample size was calculated using the formula:

$$
n=\frac{Z_{1-\alpha / 2}^{2} p(1-p)}{d^{2}}
$$

Wherein $n$ denotes number of participants, $p$ denotes expected proportion, $d$ denotes absolute precision and $1-\alpha / 2$ denotes desired level of CI. The sample size for the study objective was calculated as 113 subjects with absolute precision of $90 \%$ with an expected proportion of $0.75 \%$ at $95 \%$ CI. This study is exclusively based on male subjects who were recruited from the birth registry at the Community Health and Development (CHAD) program, Christian Medical College (CMC), Vellore, India. The participants were identified from 23 randomly selected villages from Vellore district, Tamil Nadu, South India. The contact details of the subjects born in this area were obtained from the birth registry of the CHAD program at CMC, Vellore, which has a prospective surveillance system of population-based data. Male individuals aged between 18 and 22 years were shortlisted from the database and invited for participation. The objectives of the study were explained to the participants and a cohort of 108 men without obesity were recruited with informed written consent. Individuals unwilling to participate in the study $(\mathrm{n}=5 ; 4.27 \%)$ were excluded. As the primary study was exclusively on male subjects, female subjects were not recruited as per the study design. Furthermore, individuals with prediabetes, impaired fasting glucose and dyslipidemia were excluded from participation.

All eligible participants underwent baseline medical assessment and anthropometry namely BMI and waist circumference. BMI was calculated using the formula weight $(\mathrm{kg})$ divided by height $\left(\mathrm{m}^{2}\right)$. Waist circumference was measured using a non-elastic measuring tape with the participant in standing position and in relaxed breathing state. The maximum circumference of the waist measured midway between iliac crest and lower most margin of the ribs was noted, and the hip circumference was measured at the maximum circumference of 
gluteus maximus muscle. Waist circumference $\geq 90 \mathrm{~cm}$ in males as per the Asian Indian cut-off values. ${ }^{14}$

Eligible participants were instructed to report to the study centre at 07:00 hours after an overnight fasting lasting 8 hours after supper and to avoid consuming any form of beverages in the morning. A physician examined the vital physiological parameters, prior to the start of the procedure. The participants underwent a nontracer-based 120 min HEC procedure for assessment of whole-body insulin sensitivity. In the HEC procedure, two indwelling intravenous catheters were inserted contralaterally in the veins of the antecubital fossa. In one catheter, a continuous insulin infusion was initiated and the flow rate was maintained at $40 \mathrm{mU} / \mathrm{kg} / \mathrm{min}$ using an automated infusion pump during the entire duration of the 2 hours clamp. To maintain euglycemia, $25 \%$ dextrose solution was infused and plasma glucose levels were measured by drawing blood samples from another antecubital vein, every 5 min using a bedside glucose analyzer (Analox GM-9D). The dextrose infusion rate was adjusted to maintain a stable plasma glucose concentration of $90 \mathrm{mg} / \mathrm{dL}(5 \mathrm{mmol} / \mathrm{L})$ throughout the clamp procedure. Blood samples for biochemical estimation of insulin, C-peptide and plasma glucose were drawn at baseline and at the end of the steady state phase (ie, last $30 \mathrm{~min}$ of the basal phase and the last $30 \mathrm{~min}$ of the clamp period). ${ }^{15}$ Plasma glucose levels were measured by glucose-oxidase method. Serum insulin and C-peptide levels were measured by the chemiluminescence method using diagnostic kits supplied by Siemens, on the Immulite 2000 system (Siemens Healthcare Diagnostic Products, Llanberis, Gwynedd, UK). Chemistry and Immunoassay controls supplied by Bio-Rad were used as internal precision controls (coefficient of variation (CV) $10.2 \%$ for insulin and $3.7 \%$ for C-peptide) ${ }^{13}$ Serum lipid profile including TGs was measured using an enzymebased colorimetric method in an automated analyzer (COBAS-B, 101 system, Roche Diagnostics).

The $M$ value is a measure of whole-body insulin sensitivity derived during a steady state wherein euglycemia (90 mg/dL of plasma glucose) is achieved by infusing high levels of insulin in a HEC procedure. It is calculated between 60 and $120 \mathrm{~min}$ after the start of the insulin infusion, based on the formula of DeFronzo et al. ${ }^{4}$ In this study, we applied the $M$ value cut-off value $\leq 4.7 \mathrm{mg} / \mathrm{kg}$ / min to define insulin resistance using HEC procedures. This value has been validated earlier using the results of 18 independent HEC procedures at a constant insulin infusion rate of $40 \mathrm{mU} / \mathrm{m}^{2}$ in different ethnic groups. ${ }^{16}$ Therefore, the $M$ value cut-off $\leq 4.7 \mathrm{mg} / \mathrm{kg} / \mathrm{min}$ is applicable for the current study. The following surrogate indices of insulin resistance were calculated by using specific formulae viz, LAP index: waist circumference $(\mathrm{cm})-65$ (in male subjects) $\times$ TGs $(\mathrm{mmol} / \mathrm{L}),{ }^{17}$ QUICKI: $1 /[\log$ fasting insulin $(\mathrm{mU} / \mathrm{L})+\log$ fasting glucose $(\mathrm{mg} /$ $\mathrm{dL})],{ }^{18}$ HOMA-IR: fasting glucose $(\mathrm{mmol} / \mathrm{L}) \times$ fasting insulin $(\mathrm{mU} / \mathrm{L}) / 22.5,{ }^{19}$ FG-IR: fasting glucose $(\mathrm{mg} /$ $\mathrm{dL}) /$ fasting insulin $(\mathrm{mU} / \mathrm{L}){ }^{20}$

\begin{tabular}{|c|c|}
\hline Variables $(n=108)$ & Mean \pm SD/Median \\
\hline Age (years) & $19.7 \pm 1$ \\
\hline Body masss index $\left(\mathrm{kg} / \mathrm{m}^{2}\right)$ & $19.1 \pm 2.5$ \\
\hline Waist circumference $(\mathrm{cm})$ & $70.6 \pm 5.7$ \\
\hline Waist-to-hip ratio & $0.82 \pm 0.04$ \\
\hline Waist-to-height ratio & $0.40 \pm 0.03$ \\
\hline Systolic blood pressure $(\mathrm{mm} \mathrm{Hg})$ & $118 \pm 8.5$ \\
\hline Diastolic blood pressure $(\mathrm{mm} \mathrm{Hg})$ & $76.8 \pm 6$ \\
\hline Fasting glucose (mg/dL) & $87.6 \pm 6.5$ \\
\hline Postprandial blood glucose (mg/dL) & $100.5 \pm 21.2$ \\
\hline Fasting insulin (pmol/L) & $5.2 \pm 3.6^{*}$ \\
\hline Postprandial insulin (pmol/L) & $37 \pm 29^{*}$ \\
\hline Fasting C-peptide (ng/mL) & $1.8 \pm 1.2^{*}$ \\
\hline Postprandial C-peptide (ng/mL) & $5.5 \pm 3$ \\
\hline Total cholesterol (mg/dL) & $130.7 \pm 27.6$ \\
\hline $\begin{array}{l}\text { Low-density lipoprotein cholesterol } \\
\text { (mg/dL) }\end{array}$ & $80 \pm 22.6$ \\
\hline $\begin{array}{l}\text { High-density lipoprotein cholesterol } \\
\text { (mg/dL) }\end{array}$ & $31.4 \pm 7.0$ \\
\hline Serum triglycerides (mg/dL) & $78.5 \pm 31.1$ \\
\hline
\end{tabular}

Values are presented as mean \pm SD or median (median shown with asterisk $\left.\left(^{*}\right)\right)$.

\section{Statistical analysis}

Continuous variables were summarised as mean $\pm \mathrm{SD} /$ median (minimum and maximum) values as appropriate. Spearman's correlation analysis was applied to test for significance in correlation between variables. Multivariate logistic regression analysis was applied to derive significant determinants of the LAP index. Receiver operating characteristics (ROC) analysis with area under the curve (AUC) was applied to determine the sensitivity and specificity of the cut-off value determined for LAP index. The value with optimal sensitivity and specificity was determined as cut-off value for each index. The $\mathrm{p}$ value $<0.05$ was considered to be statistically significant. STATA software (V.14.2) was used for statistical analysis.

\section{RESULTS}

The baseline characteristics of the study cohort is presented in table 1 and the $M$ value and surrogate indices of insulin sensitivity/resistance are presented in table 2.

Considering the $M$ value of the HEC procedure as the gold standard measure of insulin sensitivity, significant negative correlation was observed with the LAP index while significant positive correlation was observed with FG-IR and QUICKI (table 3).

We performed logistic regression analysis and derived BMI, low-density lipoprotein-cholesterol (LDL-C) and 
Table 2 Indices of insulin sensitivity/resistance in the study cohort $(n=108)$

\begin{tabular}{ll}
\hline Indices of insulin sensitivity/resistance & $\begin{array}{l}\text { Mean } \pm \text { SD/ } \\
\text { Median }\end{array}$ \\
\hline M value (on HEC procedure) & $10.3 \pm 3.8$ \\
Lipid accumulation product index & $25.4 \pm 13.8^{\star}$ \\
HOMA-IR & $0.9 \pm 0.8^{\star}$ \\
QUICKI (measure of insulin sensitivity) & $0.4 \pm 0.06$ \\
Fasting glucose-insulin ratio & $34.6 \pm 23.4^{*}$ \\
\hline
\end{tabular}

Values are presented as mean $\pm \mathrm{SD} /$ median (median shown with asterisk $(*))$.

HEC, hyperinsulinemic-euglycemic glucose clamp; HOMA-IR, homeostatic model assessment of insulin resistance; QUICKI, quantitative-insulin sensitivity check index.

serum TGs as significant determinants of the LAP index in this cohort. Accordingly, a decrease of 0.80 units of BMI (in $\mathrm{kg} / \mathrm{m}^{2}$ ), 0.40 units of TGs (in $\mathrm{mmol} / \mathrm{L}$ ), 0.30 $(\mathrm{mg} / \mathrm{dL})$ of LDL-C can lead to one unit decrease in the LAP index for non-obese, normoglycemic males from Southern India as shown in table 4.

ROC analysis for the LAP index derived a cut-off value $\geq 33.4$ with $75 \%$ sensitivity and $75 \%$ specificity with $10.3 \%$ and $98.7 \%$ as positive predictive value and negative predictive values, respectively for the Younden's index of 0.51 . Using the LAP index cut-off value $\geq 33.4$, we classified subjects as at risk of insulin resistance and insulin sensitive. Accordingly, the mean value of LAP index in insulin-sensitive individuals (LAP index value: $10.5, \mathrm{n}=79)$ was significantly higher $(\mathrm{p}<0.001)$ than the mean LAP index value (LAP index value: $67.1, \mathrm{n}=29$ ), in individuals classified to be at risk of developing insulin resistance in future. To compare the sensitivity and specificity of all surrogate indices, we applied a pooled ROC analysis with LAP index, FG-IR, HOMA-IR and QUICKI and plotted the AUC at 95\% CI (figure 1). The AUC was significantly higher for the LAP index when compared with HOMA-IR, QUICKI and FG-IR at 95\% CI (table 5).

\section{DISCUSSION}

This is the largest Indian study of HEC procedures done in non-obese, normoglycemic males from Southern

Table 3 Spearman's correlation of $M$ value with surrogate indices of insulin resistance/sensitivity

\begin{tabular}{lrl}
\hline $\begin{array}{l}\text { Measures of insulin sensitivity/ } \\
\text { resistance }\end{array}$ & rho & $\boldsymbol{P}$ value \\
\hline Lipid accumulation product index & -0.39 & $<0.001$ \\
\hline Fasting glucose-insulin ratio & 0.25 & $<0.01$ \\
QUICKI & 0.22 & $<0.01$ \\
HOMA-IR & 0.06 & 0.52 \\
\hline
\end{tabular}

$\mathrm{P}<0.05$ : statistically significant.

HOMA-IR, homeostatic model assessment of insulin resistance; QUICKI, quantitative-insulin sensitivity check index.
India. HEC procedures are considered the gold standard procedure to measure peripheral and hepatic insulin sensitivity. Mostly, HEC procedure-based studies done so far are on small sample sizes as it is labour intensive, technically demanding and highly expensive. ${ }^{21}$ In this study, we have shown significant correlation of the $M$ value of HEC procedure with LAP index and shown that the diagnostic accuracy of the LAP index was higher than HOMAIR, FG-IR and QUICKI as shown by ROC AUC. This proves that the LAP index is a better predictor for risk of insulin resistance in this cohort of non-obese, normoglycemic males from Southern India. Recently, a casecontrol study in middle-aged, overweight Asian Indians from India by Ray et al has shown that LAP index is a better predictor of metabolic syndrome when compared with BMI and waist circumference. ${ }^{11}$ We compared the observations of Ray et $a l^{11}$ and the current study. The former study derived a LAP index cut-off value of $\geq 38.05$ with $76.4 \%$ and $91.1 \%$ specificity in a cohort of subjects with obesity ${ }^{11}$ whereas in the current study, we derived a lower cut-off value $\geq 33.4$ with $75 \%$ sensitivity and $75 \%$ specificity. It may be noted that the case control study by Ray et al included subjects with obesity with metabolic syndrome, ${ }^{11}$ in contrast to our study exclusively on non-obese, normoglycemic males. Specifically, the mean waist circumference and serum TG levels in the current study were significantly lower than that of Ray et $a l^{11}$ thus leading to difference in cut-off values of the LAP index between two studies. In the current study, BMI, LDL and TGs were derived as significant determinants of the LAP index. However, the study by Ray et al in Indians did not include biochemical variables and therefore derived BMI and waist circumference as independent determinants of LAP index. Furthermore, it did not correlate the LAP index with other surrogate indices of fasting insulin resistance or the $M$ value of HEC procedure, whereas the current study has accomplished this lacuna.

Another Indian study has shown significantly higher value $(p<0.001)$ of the LAP index in patients with psoriasis when compared with normal subjects. The LAP index was significantly higher $(\mathrm{p}<0.05)$ in the moderate-tosevere psoriasis group as compared with the mild psoriasis group. ${ }^{22}$ In a population-based study from Gujarat, it has been shown that the LAP index showed superior diagnostic accuracy for metabolic syndrome in asymptomatic subjects with normoglycemia aged between 18 and 79 years. The ROC AUC for the LAP index was 0.82 for a cut-off value $34.7 .{ }^{10}$ In comparison to the same, the LAP index cut-off value derived in our study $(\geq 33.4)$ is nearly similar with an ROC AUC of 0.71 . However, the study by Joshi et $a l^{10}$ was not based on HEC procedures unlike the current study.

Internationally, the LAP index has been studied in other ethnic groups. An earlier study in 768 elderly Caucasians with normoglycemia had shown the LAP index as a superior predictor of metabolic syndrome. Specifically, the cut-off value $(\geq 51.82)$ for LAP index showed higher sensitivity $(0.85)$ and specificity $(0.85) .^{23}$ 
Table 4 Logistic regression analysis for significant determinants of the lipid accumulation product index

\begin{tabular}{llllll}
\hline Predictors & Beta-coefficient & OR & SE & 95\% CI & $\boldsymbol{P}$ value \\
\hline Body mass index $\left(\mathrm{kg} / \mathrm{m}^{2}\right)$ & -0.80 & 0.45 & 0.17 & 0.32 to 0.63 & $<0.001$ \\
Serum triglycerides $(\mathrm{mg} / \mathrm{dL})$ & -0.40 & 1.0 & 0.01 & 0.93 to 0.98 & $<0.001$ \\
Low-density lipoprotein-cholesterol $(\mathrm{mg} / \mathrm{dL})$ & -0.30 & 1.0 & 0.01 & 0.95 to 0.99 & $<0.01$ \\
\hline
\end{tabular}

$\mathrm{P}<0.05$ : statistically significant.

$\mathrm{Cl}$, confidence Interval; OR, odds ratio; SE, standard error.

The LAP index has also been applied as a surrogate measure of metabolic syndrome in a Chinese cohort by $\mathrm{Li}$ et al. On comparing the LAP index cut-off values of $\mathrm{Li}$ et $a l^{17}$ and Teverna et $a l^{23}$ we report a significantly lower cut-off value $\geq 33.4$ with $75 \%$ sensitivity and $75 \%$ specificity for the Indian population. The differences can be evidently attributed to ethnic variations and differences in age groups between the studies.

The LAP index has been validated recently in a larger cohort of Chinese subjects $(n=711)$ with type 2 diabetes mellitus in comparison to VAI and waist circumference-TG index. Among these indices, the highest AUC was observed for the LAP index cut-off value $\geq 44.0$ in both male and female subjects. Furthermore, LAP index was found to be a simple and superior indicator of metabolic syndrome in the study cohort. ${ }^{24}$ In comparison to the study by Ma $e t a l^{24}$ the LAP index cut-off value to detect risk of insulin resistance in the current study is significantly lower, which could be attributed to the differences in ethnicity, age, gender and physiological status of the cohorts between the two studies. Wiltgen et $a l$, derived a LAP index cut-off value of $\geq 34.5$ (sensitivity $84 \%$; specificity $79 \%$ ) in a cohort of Brazilian women $(\mathrm{n}=95)$ with metabolic syndrome and observed significant

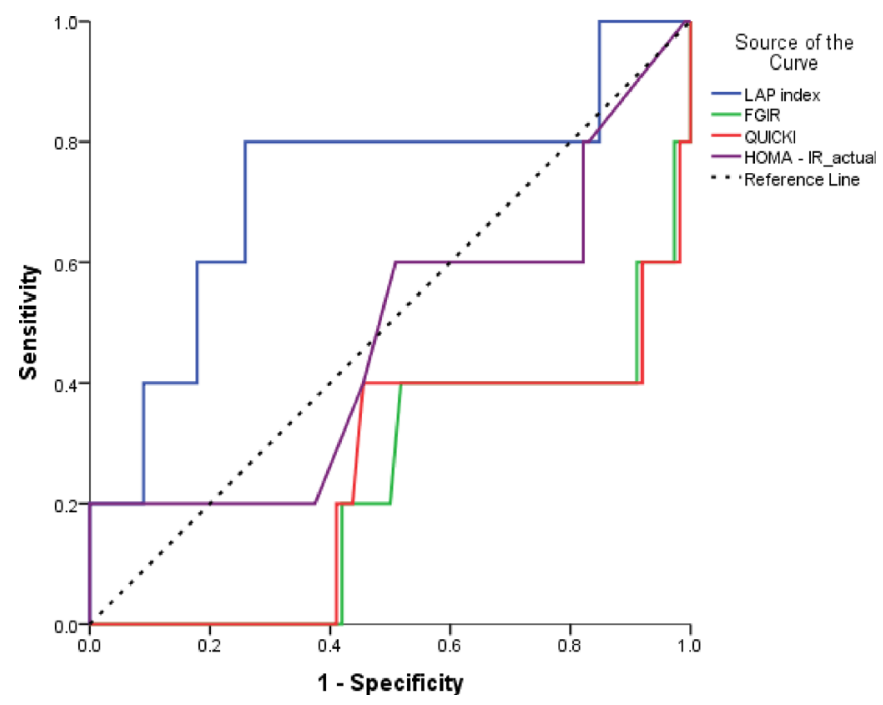

Figure 1 Receiver operating characteristic area under curve with sensitivity, in \% (on Y-axis) and 1-specificity in $\%$ (on X-axis) at 95\% CI for the LAP index, FG-IR, QUICKI and HOMA-IR. FG-IR, fasting glucose-insulin ratio; HOMA$I R$, homeostatic model of assessment of insulin resistance; LAP index, lipid accumulation product index; QUICKI, quantitative-insulin sensitivity check index. positive correlation of the LAP index with HOMA-IR and waist circumference. ${ }^{25}$ However, the authors suggested that the LAP index should be validated against the glucose clamp procedure in Asian Indians. The current study has accomplished this milestone and has shown significant correlation of the LAP index with QUICKI in a cohort of non-obese, normoglycemic Asian Indian males.

South Asians feature higher prevalence of lipoprotein abnormalities and have a twofold to threefold higher risk of developing cardiovascular disease ${ }^{26}$ and $\mathrm{T}_{2} \mathrm{DM}^{27}$ as compared with white Caucasians. ${ }^{28}$ It is important to note the presence of insulin resistance even in non-obese, normoglycemic Asian Indians, ${ }^{29}$ due to atherogenic dyslipidemia, specifically chronic hypertriglyceridemia. ${ }^{26}$ Hypertriglyceridemia results from increased fatty acid synthesis and decreased fatty acid oxidation, which in turn leads to increased hepatic secretion of very lowdensity lipoprotein cholesterol. ${ }^{30}$ As an effect, there is an increased hepatic influx of non-esterified, free fatty acids (FFA), mediated by lipoprotein lipase enzyme, ${ }^{31}$ resulting in the onset of peripheral and hepatic insulin resistance. ${ }^{32}$ In the pancreas, the influx of FFAs leads to decreased beta cell function and insulin resistance, irrespective of body weight. ${ }^{33}$ At the adipose tissue, the influx of FFAs and insulin resistance results in decreased glucose metabolism and impaired glycerol synthesis, irrespective of body weight and age. ${ }^{34}$ In such a scenario, it is imperative to screen Asian Indians of representative sample sizes for metabolic syndrome, using novel surrogate indices based on TGs. The results of this HEC-based study evidenced superior performance of the LAP index which is cost-effective as compared with HOMA-IR and QUICKI which rely on plasma insulin levels thus proving its utility in low-cost clinical settings. The LAP index has high feasibility value, as it does not necessarily require an overnight fasting state making it an ideal index for use in epidemiological studies.

\section{Limitations of the study}

This study is cross-sectional in an exclusive cohort of non-obese, normoglycemic males from Southern India, which limits the applicability of the study observations to the cohort. Thus, the need to test this index in females becomes imperative. In addition, gender-specific studies on LAP index, across age and BMI groups need to be done. Furthermore, as this study is on normoglycemic subjects, specific cut-off values for LAP index in patients with T2DM are required. Nevertheless, the study observations 
Table 5 Receiver operating characteristic area under curve (AUC) for surrogate indices

\begin{tabular}{lllllll}
\hline Indices of insulin sensitivity/resistance & AUC & Cut-off value & Sensitivity (\%) & Specificity (\%) & 95\% CI & SE \\
\hline Lipid accumulation product index & 0.72 & $\geq 33.4$ & 75 & 75 & 0.50 to 0.94 & 0.14 \\
HOMA-IR & 0.55 & $\geq 0.75$ & 75 & 46 & 0.26 to 0.85 & 0.15 \\
QUICKI & 0.31 & $\geq 0.40$ & 50 & 50 & 0.04 to 0.57 & 0.13 \\
Fasting glucose-insulin ratio. & 0.29 & $\geq 23.4$ & 50 & 48 & 0.04 to 0.53 & 0.12 \\
\hline
\end{tabular}

$\mathrm{Cl}$, confidence interval; HOMA-IR, homeostatic model assessment of insulin resistance; QUICKI, quantitative-insulin sensitivity check index; SE, standard error.

based on HEC procedure in non-obese normoglycemic, Asian Indian males are important and can be validated in clinical settings and through population-based studies from different parts of India.

Acknowledgements We thank the participants and field workers involved in this study. Financial support offered by the Department of International Health, University of Copenhagen, Denmark for the primary study from this cohort is gratefully acknowledged. However, the current study on LAP index is independent of financial support.

Contributors SAS and RD conceptualized the study. GR analyzed the data and interpreted it. NT and MPI performed HEC procedures. AJ and GF performed biochemical assays. SAS reviewed the data and drafted the manuscript. RD and NT contributed to the discussion and reviewed the manuscript.

Funding The authors have not declared a specific grant for this research from any funding agency in the public, commercial or not-for-profit sectors.

Competing interests None declared.

Patient consent for publication Not required.

Ethics approval Institutional research board and human ethics committee of Christian Medical College, Vellore, India (Research Committee Minute Number: 13348/RETRO/28/08/2020 of Christian Medical College, Vellore, India).

Provenance and peer review Not commissioned; externally peer reviewed.

Data availability statement All data relevant to the study are included in the article. All data relevant to the study are included in the article as tables, text and figures.

Open access This is an open access article distributed in accordance with the Creative Commons Attribution Non Commercial (CC BY-NC 4.0) license, which permits others to distribute, remix, adapt, build upon this work non-commercially, and license their derivative works on different terms, provided the original work is properly cited, appropriate credit is given, any changes made indicated, and the use is non-commercial. See: http://creativecommons.org/licenses/by-nc/4.0/.

\section{ORCID iDs}

Riddhi Dasgupta http://orcid.org/0000-0003-0838-1015

Nihal Thomas http://orcid.org/0000-0002-4614-9519

\section{REFERENCES}

1 WHO. Ncds | major NCDS and their risk factors. Available: http:// www.who.int/ncds/introduction/en/ [Accessed 10 Sep 2020].

2 Misra A, Khurana L. Obesity-related non-communicable diseases: South Asians vs white Caucasians. Int J Obes 2011;35:167-87.

3 Liew C-F, Seah E-S, Yeo K-P, et al. Lean, nondiabetic Asian Indians have decreased insulin sensitivity and insulin clearance, and raised leptin compared to Caucasians and Chinese subjects. Int $J$ Obes Relat Metab Disord 2003:27:784-9.

4 DeFronzo RA, Tobin JD, Andres R. Glucose clamp technique: a method for quantifying insulin secretion and resistance. Am J Physiol 1979;237:E214-23.

5 Singh B, Saxena A. Surrogate markers of insulin resistance: a review. World J Diabetes 2010;1:36-47.

6 Venkatesan P, Tiwari A, Dasgupta R, et al. Surrogate measures of insulin sensitivity when compared to euglycemic hyperinsulinemic clamp studies in Asian Indian men without diabetes. J Diabetes Complications 2016;30:287-91.
7 Kahn HS. The "lipid accumulation product" performs better than the body mass index for recognizing cardiovascular risk: a populationbased comparison. BMC Cardiovasc Disord 2005;5:26.

8 Fiorentino TV, Marini MA, Succurro E, et al. Relationships of surrogate indexes of insulin resistance with insulin sensitivity assessed by euglycemic hyperinsulinemic clamp and subclinical vascular damage. BMJ Open Diabetes Res Care 2019;7:e000911.

9 Long J, Li L, Wang F, et al. Screening for a simple and effective indicator of insulin resistance in Chinese Reproductive-Aged women, with the insulin clamp technique as a reference. Metab Syndr Relat Disord 2019;17:423-9.

10 Joshi H, Shah K, Patel P, et al. Novel indexes for diagnosing metabolic syndrome in apparently healthy Gujarati Asian Indians: a cross-sectional study. QJM 2016;109:717-22.

11 Ray L, Ravichandran K, Nanda SK. Comparison of lipid accumulation product index with body mass index and waist circumference as a predictor of metabolic syndrome in Indian population. Metab Syndr Relat Disord 2018;16:240-5.

12 Ramdas Nayak VK, Nayak KR, Vidyasagar S, et al. Predictive performance of traditional and novel lipid combined anthropometric indices to identify prediabetes. Diabetes Metab Syndr 2020;14:1265-72.

13 Thomas N, Grunnet LG, Poulsen P, et al. Born with low birth weight in rural southern India: what are the metabolic consequences 20 years later? Eur J Endocrinol 2012;166:647-55.

14 Misra A, Chowbey P, Makkar BM, et al. Consensus statement for diagnosis of obesity, abdominal obesity and the metabolic syndrome for Asian Indians and recommendations for physical activity, medical and surgical management. J Assoc Physicians India 2009;57:163-70.

15 Thomas N, Grunnet LG, Poulsen P, et al. Born with low birth weight in rural southern India: what are the metabolic consequences 20 years later? Eur J Endocrinol 2012;166:647-55.

16 Bergman RN, Finegood DT, Ader M. Assessment of insulin sensitivity in vivo. Endocr Rev 1985;6:45-86.

17 Li R, Li Q, Cui M, et al. Clinical surrogate markers for predicting metabolic syndrome in middle-aged and elderly Chinese. J Diabetes Investig 2018;9:411-8.

18 Chen H, Sullivan G, Quon MJ. Assessing the predictive accuracy of QUICKI as a surrogate index for insulin sensitivity using a calibration model. Diabetes 2005;54:1914-25.

19 Katsuki A, Sumida Y, Gabazza EC, et al. Homeostasis model assessment is a reliable indicator of insulin resistance during followup of patients with type 2 diabetes. Diabetes Care 2001;24:362-5.

20 Legro RS, Finegood D, Dunaif A. A fasting glucose to insulin ratio is a useful measure of insulin sensitivity in women with polycystic ovary syndrome. J Clin Endocrinol Metab 1998;83:2694-8.

21 Borai A, Livingstone C, Kaddam I, et al. Selection of the appropriate method for the assessment of insulin resistance. BMC Med Res Methodol 2011;11:158.

22 Ganguly S, Ray L, Kuruvila S, et al. Lipid accumulation product index as visceral obesity indicator in psoriasis: a case-control study. Indian J Dermatol 2018;63:136-40.

23 Taverna MJ, Martínez-Larrad MT, Frechtel GD, et al. Lipid accumulation product: a powerful marker of metabolic syndrome in healthy population. Eur J Endocrinol 2011;164:559-67.

24 Ma C-M, Lu N, Wang R, et al. Three novel obese indicators perform better in monitoring management of metabolic syndrome in type 2 diabetes. Sci Rep 2017;7:9843.

25 Wiltgen D, Benedetto IG, Mastella LS, et al. Lipid accumulation product index: a reliable marker of cardiovascular risk in polycystic ovary syndrome. Hum Reprod 2009;24:1726-31.

26 Bilen O, Kamal A, Virani SS. Lipoprotein abnormalities in South Asians and its association with cardiovascular disease: current state and future directions. World J Cardiol 2016;8:247-57. 
27 Ding C, Chan Z, Chooi YC, et al. Regulation of glucose metabolism in nondiabetic, metabolically obese normal-weight Asians. Am J Physiol Endocrinol Metab 2018;314:E494-502.

28 Dodani S. Excess coronary artery disease risk in South Asian immigrants: can dysfunctional high-density lipoprotein explain increased risk? Vasc Health Risk Manag 2008;4:953-61.

29 Petersen KF, Dufour S, Feng J, et al. Increased prevalence of insulin resistance and nonalcoholic fatty liver disease in Asian-Indian men. Proc Natl Acad Sci U S A 2006;103:18273-7.

30 Cohn JS, Patterson BW, Uffelman KD, et al. Rate of production of plasma and very-low-density lipoprotein (VLDL) apolipoprotein C-III is strongly related to the concentration and level of production of VLDL triglyceride in male subjects with different body weights and levels of insulin sensitivity. J Clin Endocrinol Metab 2004;89:3949-55.

31 Raz I, Eldor R, Cernea S, et al. Diabetes: insulin resistance and derangements in lipid metabolism. cure through intervention in fat transport and storage. Diabetes Metab Res Rev 2005;21:3-14.

32 Boden G. Obesity, insulin resistance and free fatty acids. Curr Opin Endocrinol Diabetes Obes 2011;18:139-43.

33 Itoh Y, Kawamata Y, Harada M, et al. Free fatty acids regulate insulin secretion from pancreatic beta cells through GPR40. Nature 2003;422:173-6.

34 Mostaza JM, Vega GL, Snell P, et al. Abnormal metabolism of free fatty acids in hypertriglyceridaemic men: apparent insulin resistance of adipose tissue. J Intern Med 1998;243:265-74. 